\title{
Research on regimen furniture based on the theory that acupuncture can cure chronic diseases
}

\author{
Xinyan Yang ${ }^{1}$, Zhongfeng Zhang ${ }^{2}$ \\ ${ }^{1}$ Central South University of Forestry and Technology, Changsha, Hunan, 410004, China \\ ${ }^{2}$ Central South University of Forestry and Technology, Green furniture engineering technology research center of State Forestry and \\ grassland administration, Hunan Provincial Green House Engineering Technology Research Center, Changsha, Hunan, 410000, China
}

\begin{abstract}
In recent years, the development of the economy has prompted the furniture industry to diversify, and massage furniture is constantly emerging. Many massage products are relatively limited in design, and prone to problems such as general body massage, acupuncture points that are not symptomatic, and popularization of the use of the crowd. As a result, massage products can only solve "temporary problems" and do not cure the symptoms. This article summarizes and researches on the previous achievements of acupuncture points for chronic diseases. The acupoint-dense area is refined and designed as a machine with a grid-like massage path. The treatment effect is enhanced by the "point-to-point" method, so as to achieve the concept of "prevent diseases" in Chinese medicine. This type of furniture not only has health functions, but also increases the added value of the furniture. Health-care furniture can increase the added value of furniture and design new directions for the furniture industry.
\end{abstract}

\section{Introduction}

Driven by artificial intelligence, the furniture industry is moving towards intelligence. There are few theories about health-care furniture at home and abroad. Most of them are massage chairs and massage beds. Many furniture with massage and health care functions are limited and the treatment effect is not satisfactory. This article researches and summarizes the cases of traditional Chinese medicine acupoint treatment of chronic diseases, and proposes a design method of health-care furniture to solve problems such as insufficient treatment and too general massage.

\section{Acupoints can cure chronic disease theory}

\subsection{Chronic disease concepts and types}

Chronic. It refers to a general term for diseases that are not a source of infection and cause damage to part of the body or organs due to long-term accumulation of toxins, not just a certain disease. If not treated in time, it can cause damage to the heart, brain, and organs, which can seriously endanger life. According to the "China Health Survey Report", in 2012, the number of deaths due to chronic diseases in China accounted for $86.6 \%$ of the total deaths. Among them, 22\% of middle-aged people died of cardiovascular and cerebrovascular diseases. The elderly have a high prevalence of chronic diseases and there are many factors such as complications in clinical practice. In addition, the obese population will reach 325 million and will double in the next 20 years. Recently, there have been frequent cases of sudden death of young people at work, and many chronic (acute) diseases have gradually become younger. In Wang Zhaoxin's research, it was found that diabetes, blood, and endocrine system diseases are being transferred to young people aged 3050.

According to the body's functional system, chronic diseases can be divided into: respiratory diseases, circulatory system diseases, urinary system diseases, blood system diseases, digestive system diseases, metabolic system diseases, rheumatic system diseases, and neurological diseases. Using literature collation methods, sort out the acupuncture points in the clinical cases of traditional Chinese medicine treatment of the above chronic diseases.

\subsection{Acupoints for the treatment of chronic diseases}

2.2.1. Respiratory disease. As shown in Table. 1, according to Guo's winter disease and summer acupoint application therapy, the proportion of respiratory diseases is the highest accounting for $34.73 \%$ of the total in the 60-74 years old, and common such as chronic bronchitis, asthma. "Su Wen · Cough Theory" mentioned, "People who treat organs, treat their acupoints." Choosing the right acupuncture points can help treat and prevent this type of disease. In clinical practice, multiple 
back-shu points, lung-shu points, Dazhui and Tiantu points are often used. Chronic cough and asthma bronchitis: Feishu, Pishu, Weishu, Shenshu, Zhishi and Zhongfu. According to Hu Zhiguang's treatment of 61 cases of bronchial asthma patients with acupuncture at Dazhui, Dingchuan, Fengmen, Feishu, Tsusanli, Sanyinjiao, and Kongkong, the efficiency reached 81.97\%. According to Zhang Yali's records: cold: desirable lung, inner nose, lower screen tip, cough: bronchus, lung, Shenmen, asthma: asthma, lung, lower foot end, lower screen tip. Commonly used for cardiopulmonary treatment: The urinary bladder channel of Foot-Taiyang, Gaohuangshu, Du shu. Zhao Zhihong treated 200 cases of chronic bronchitis for three consecutive years with a total efficiency of $85.5 \%$ by taking acupuncture points such as Feishu, Xinshu, Geshu, Fengmen, Shenshu, Jueyinshu, Tiantu, Dazhui, Shanzhong, Gaohuang, Zhongwan, Zhongfu, Neiguan, Dingchuan, etc. Hu Ping chooses Feishu, Pishu, Shenshu, Gaohuang with drugs to achieve a total treatment efficiency of $86.5 \%$. Table 1 are acupuncture points for treating the disease.

Table. 1 Gender and age composition of respiratory diseases

\begin{tabular}{c|c|c|c|c|c|c|}
\hline \multicolumn{9}{|c|}{ Sex, age composition } \\
\hline \multirow{2}{*}{ generation } & \multirow{2}{*}{ cases } & total ratio & \multicolumn{2}{|c|}{ men } & \multicolumn{2}{c|}{ women } \\
\cline { 4 - 8 } & & & cases & ratio & cases & ratio \\
\hline $18-44$ & 322 & 24.52 & 144 & 27.27 & 178 & 22.67 \\
\hline $45-59$ & 366 & 27.88 & 113 & 21.4 & 253 & 32.23 \\
\hline $60-74$ & 456 & 34.73 & 179 & 33.9 & 277 & 35.29 \\
\hline$<75$ & 169 & 12.87 & 92 & 17.43 & 77 & 9.81 \\
\hline total & 1313 & 100 & 528 & 100 & 785 & 100 \\
\hline
\end{tabular}

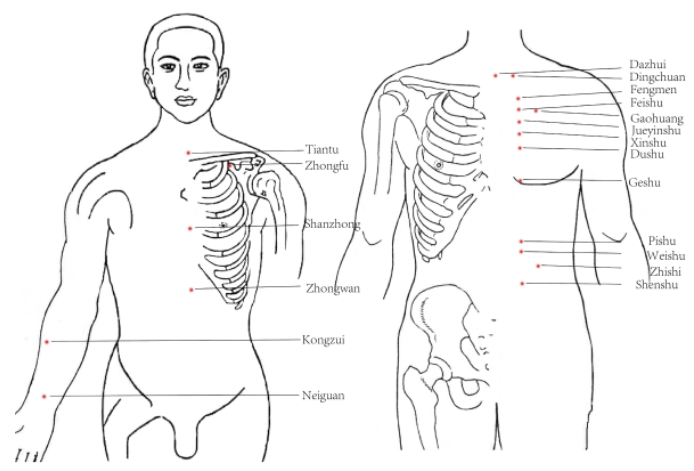

Figure. 1 Acupuncture points for treating the respiratory disease

2.2.2. Circulation system disease. The main types of these diseases are: chronic heart failure, coronary disease, cardiomyopathy, chronic atrial fibrillation, cardiac insufficiency. The word "thoracic obstruction" appears in the Internal Canon of Medicine, which also refers to the symptoms of coronary disease and angina pectoris. Although it is not clearly defined, the term includes all chest obstructive diseases. In the study of coronary heart disease, Li Liuyi divided this type of disease into the following categories: cardialgia, pectoralgia and septal pain; thoracic obstruction, heart palsy; reversal heart pain, angina pectoris; stroke heartache, chronic stomachache; Heart and spleen pain, Heart cold disease, Cardiac hernia.To treat this type of disease, acupoints are often taken at the wrists and ankles, such as Daling Point, Yuji Point, Taiyuan, Taibai, Jinggu, Kunlun. According to the fixed acupuncture points summarized by Li's: Jujue, Xinshu, Shendao, Neiguan, Shanzhong, Jueyinshu, Zhiyang, and Ximen.

The study found that the longer the compression time and the greater the intensity, the better the effect.
After comparing the therapeutic effects of the two groups, Li Jie concluded that massage Shenjue point has a better effect on chronic cardiac insufficiency and is worthy of clinical promotion. In his research, He Zhiming explained that treatment of coronary heart disease focuses on both the root and branch aspects and take acupuncture points such as Gongsun, Neiguan, Lieque, Zhaohai, Waiguan, Tsulinch'i Houxi, Shenmai. Because of different clinical conditions, adding individual acupoints on the basis can improve the treatment effect, such as acupoint Spleen Shu, Shanzhong, Qihai, Tsusanli, Shenshu for deficiency of vital energy; acupoint Sanyinjiao, Taichong, Fuliu, Renzhong for deficiency of Yin; acupoint Dazhui, Guanyuan, Suliao for Cardioyangpenia; acupoint Gongsun, Neiguan, Geshu,

Rangu, Fenglong, Yinlingquan for coagulation of blood; acupoint Rangu, Fenglong, Yinlingquan, Spleen Shu for phlegm obstruction. Liu Wanning concluded that acupuncture Quchi, Tsusanli, Taichong, Shenmen, Ganshu, Guanyuan, and Neiguan had significant reduction effects on systolic blood pressure. 


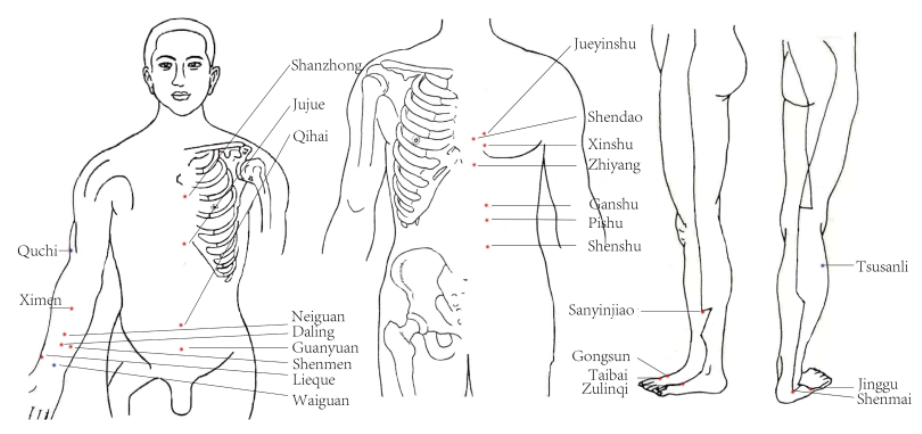

Figure. 2 Acupuncture points for treating the circulation system disease

2.2.3. Urinary system disease. The system diseases include: chronic renal failure, chronic glomerulonephritis, and nephrotic syndrome. Nephrotic syndrome belongs to the category of "edema" in the theory of traditional Chinese medicine. Depending on the clinical situation, it is sometimes related to "turbid urine", "Lumbago", "consumption" and "obstruction and rejection".Su Wen. Water-heat Acupuncture Theory" mentioned, "Those who are brave and hardworking sweat out of the kidney and then meets the wind, do not enter the viscera inside and do not go beyond the skin outside, it is said to be bloated, which is inherent in the kidney. Its name is Feng Shui." Pan Yuting summarizes the pathogenesis of nephrotic syndrome in the past doctors: Deficiency of the five internal organs, including lung, spleen and kidney disorders, deficiency of spleen and kidney, deficiency of kidney, and spleen and stomach disorders; evil pathogen is caused, including rheumatism, endoretention of damp heat and stagnated heat in xuefen. In recent years, due to irregular personal habits of modern people, the incidence of chronic kidney disease has increased. Factors such as obesity, drug abuse, and hypertension can increase the risk of kidney disease. According to Wang Huan, the acupuncture points of this type of disease are summarized: Chronic nephritis: Shenshu, Tsusanli, Yongquan; Chronic renal failure: Shenshu, Qihai, Shuifen, Sanyinjiao; Nephrotic syndrome : Shenjue, Double renal pelvis, Shuifen,Shuidao, Sanjiaoshu, Weiyang, Yinlingquan, Bladder Shu, Gaohuangshu; Diabetes mellitus: Taichong, Taixi,Tsusanli, Huantiao, Shangjuxu, Yishe, Qihai, Shenshu, Small intestine shu, Rangu, Xingjian. Acupuncture points can be used to improve chronic kidney disease such as nocturnal clearance, lumbar debility, fatigue and lack of strength etc.



Figure. 3 Acupuncture points for treating the urinary system disease

2.2.4. Hematological disease. Chronic diseases of the hematological diseases have various causes of anemia. Anemia is a clinical syndrome with a lower red blood cell capacity. It is not an independent disease, but may be an important clinical manifestation of a basic or sometimes more complicated disease. "Su wen abdominal theory" mentioned, "People who have a full chest and threatened disease should be protected from food...the limbs are clear, the eyes are dazzled...and the disease name is blood exhaustion," "Medical science: medical science or questions" mentioned, "The cause of weakness is from the kidneys." means kidney deficiency does not store essence and no hematopoietic effect. Therefore, from the perspective of traditional Chinese medicine, chronic aplastic anemia lies in the deficiency of the spleen and kidney and is mainly in the kidney. In addition to traditional Chinese herbal enema and way of pasting therapy, it is also necessary to massage acupoints. Articles on the treatment of chronic aplastic anemia by external treatment of Chinese medicine first appeared in the 1960s, Zhao Guixiang selected acupoints such as Yintang, Xuehai, Yuji, and Dazhui to achieve $90 \%$ of the therapeutic effect. Yang Zhiwen used internal administration plus acupoints such as Mingmen, Shenshu, Pishu, Tsusanli and other acupoints to treat 36 cases of the disease with an efficiency of $86.1 \%$. On the basis of researching her tutor to treat leukopenia, Wang Hui concluded that anemia due to leukopenia is related to "Weiyang". Massage Qi Hai, Guan Yuan, Tsusanli and other acupuncture points can replenish vital energy and treat clinical manifestations such as white face, fatigue, sweating, and susceptible pathogen. 


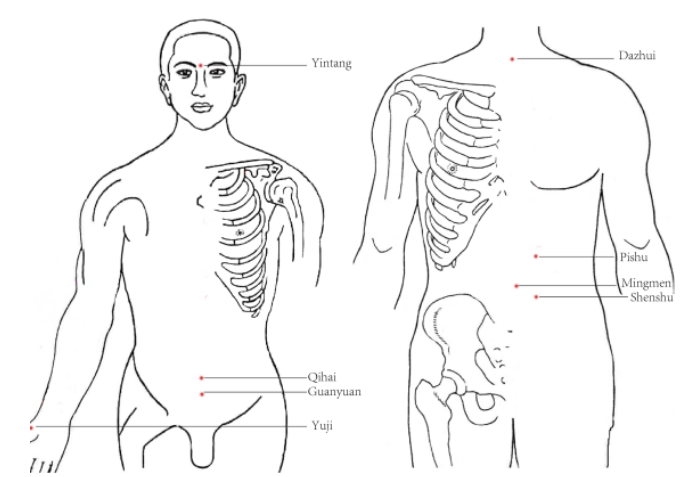

Figure. 4 Acupuncture points for treating the hematological disease

2.2.5. Digestive system disease. Cirrhosis, chronic hepatitis, and peptic ulcer are the main clinical manifestations of this system disease. Cirrhosis is the last stage of the development of liver fibrosis, with mild symptoms at the early stage and a series of complications due to liver insufficiency, such as hepatic encephalopathy, peritonitis, and hepatorenal syndrome. ascites due to cirrhosis have become a worldwide refractory disease. $\mathrm{Xu}$ Jie summarized that kidney deficiency is caused by spleen deficiency, which affects liver and kidney function by a long time and eventually leads to excessive splanchnic fluid and swelling. In his research, Shenjue, Qimen, Zhangmen were selected for the application treatment. There are 30 acupoints related to the treatment of cirrhosis in Duan Yinghua's statistical literature: Tsusanli, Ganshu, Qimen, Shenjue, Sanyinjiao, Taichong, Zhangmen, Yanglingquan, Pishu, xiahe and bahui are commonly used for conditioning viscera. Peptic ulcer is a gastrointestinal dysfunction, while hepatogenic gastrointestinal insufficiency is a dysfunction in urinary tract secretion, absorption and exercise circulation caused by hepatic insufficiency. Deng Jingjing mentioned the daily electroacupuncture at Tsusanli, Sanyinjiao and Taichong after observing the acupuncture effect of 40 gastrointestinal patients with cirrhosis. These three acupoints are one of the "Jin San Acupuncture" created by Lingnan acupuncture expert Professor Jin Rui. Zusanli is the point of stomach meridian and Sanyinjiao is the point of spleen meridian. Combination of two points can relieve liver and qi.

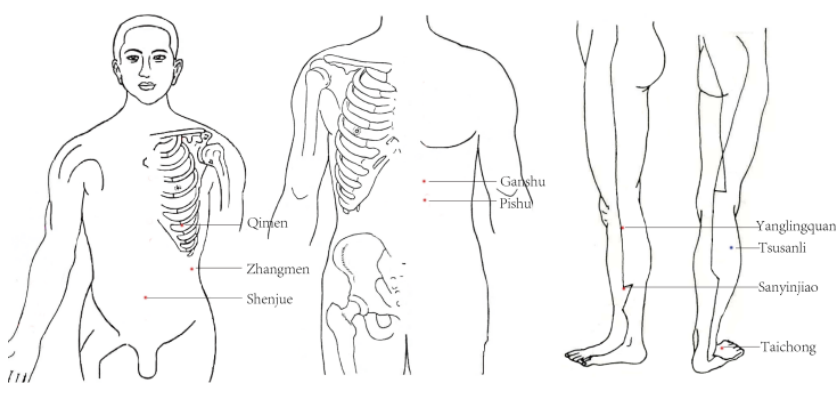

Figure. 5 Acupuncture points for treating the digestive system disease

2.2.6. Rheumatic disease. Rheumatoid arthritis is a systemic autoimmune disease mainly characterized by erosive arthritis, polyarticular synovitis and extraarticular lesions. Chinese medicine believes that this type of disease is the clinical manifestations of wind, cold, dampness, heat and other evil gases invading the human body, resulting in poor flexion and extension. Although various famous medical experts have different acupuncture methods and acupoints, their total efficiency is quite high. Such as Yin Baishun's acupuncture therapy, acupoints of Great Slaughter, Ganshu, Shenshu, bladder Shu, Weizhong, Kunlun, Waiguan, Fengshi, Xuehai, Taixi, Sanyinjiao, achieving a total efficiency of $96.8 \%$. Wang Xihua used fire acupuncture therapy, taking Tianying, Jiaji, partial acupoints on the extremities, and Zhongwan, Qihai, Guanyuan, Tsusanli, Huantiao, Yinlingquan, Yangling,Bafeng, Baxie, reached the total efficiency is $97 \%$.Guo Aihua used catgut implantation at acupoint to take Hegu, partial calendar, Quchi, Zusanli, Jiexi, Huantiao to treat 6 cases of the disease. Sun Wenshan taking points Ganshu, Pishu, Shenshu, Mingmen, Quchi,Tsusanli, Taixi, and local joints acupoints. The symptoms of half-month treatment were significantly reduced. Tan Sanchun taking points Shenshu, Yingu, Taixi, Guanyuan, Xuanzhong, Geshu, Xuehai, Fengchi, Yinlingquan, Tsusanli and other local points. Zhang Kuo selected 143 acupoints to treat this kind of disease, and summarized the most commonly used acupoint for different parts. Based on the above, the common points are summarized as follows.
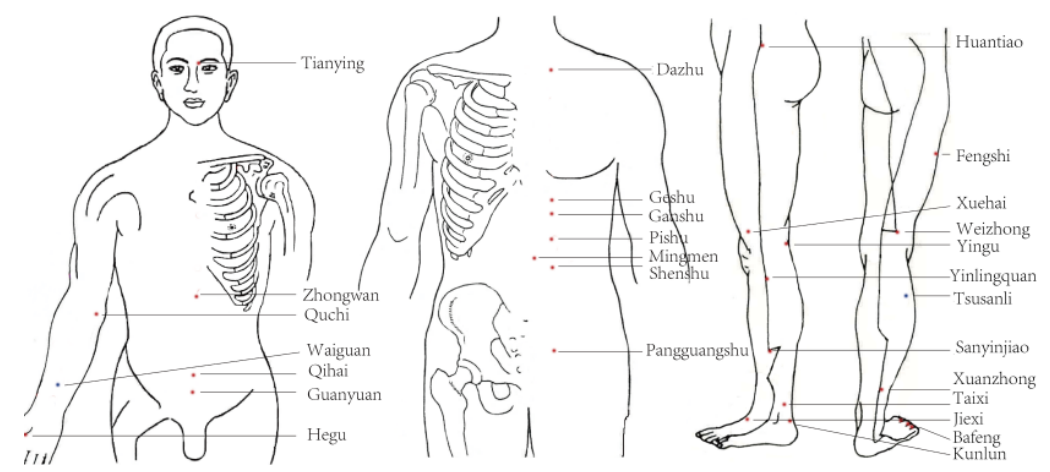

Figure. 6 Acupuncture points for treating the rheumatic disease 


\section{The furniture of regimen}

A furniture of regimen is evolved according to the development process of human beings. China's bed was produced in Yin Shang dynasty and began to sat on the chair in Song dynasty. When modern design began, electric massage tables for nursing homes appeared in 1910, the first massage bed appeared in the late 1920s and then various massage products appeared in the 1940s. In 2002, health-care furniture appeared abroad such as temperature-adjustable beds, hypnotic and wake-up beds, magnetic therapy chairs, mandatory brain chairs, and so on. Chinese traditional furniture pays attention to the health function of materials, such as red sandalwood for sleeping, rosewood for sitting and lying, carry sea yellow with oneself, chicken wing wood for daily use, ebony for decoration, camphor for decorate. In 2009, Wei Qingqing specifically discussed the definition and innovative methods of health care furniture. It is a piece of furniture that uses high technology and new materials to meet people's various health needs. Zhang Genlei studied rehabilitation furniture in 2018 with reference to the term "rehabilitation" in biomedicine and "rehabilitation landscape" in environmental psychology. The development direction of the furniture industry is more diversified and the furniture of regimen facing specific illnesses can be developed.

\subsection{The concept of regimen}

"Regimen" was proposed by American doctor Dr. Halbert Dunn in 1961 and interpreted as "wellbeing" and "fitness". Other foreign scholars have successively proposed regimen-related theories. For example, Adams proposed four basic points for regimen: multidimensional, relative subjectivity, maintenance-oriented, and balanced. In addition, septuple space about regimen proposed by Puczko and Bachvarov. In China, the concept of regimen was first seen in the "Chuang-tzu's inner chapter". Regimen has the meaning of maintenance, life and growth and recuperation. It is an activity to achieve physical fitness through various methods such as fostering spirit, adjusting diet, practicing body shape, and adapting to cold temperatures. the most common ways are acupuncture, massage, diet health care.

$\mathrm{Li} \mathrm{Li}$, a domestic scholar, defined the differences in the connotation of regimen between China and the West in 2006 and found that differences were mainly due to their View of nature and way of thinking. The sprouting of health-care ideas in China appeared in the Yin and Shang dynasties, and gradually developed during the Western Zhou Dynasty until the Spring and Autumn Period. According to "Zuo Zhuan", a treatment incident of a doctor from Qin dynasties have shown that people noticed the relationship between daily living and health. In the same year, Liang Yanli pointed out a series of Taoist health regimens. Yang Jianhua studied the Confucian health philosophy. After the precipitation of history. Chinese traditional health culture has gradually developed into a culture of Confucianism, Taoism, Buddhism, and medicine.

\subsection{Elements of furniture design of regimen}

The elements of furniture of regimen design are as follows:

Swing and rotation: The common postures of the human body are standing, sitting, and lying. In order to meet different postures, the structure of furniture of regimen must have transformations such as swing, rotation and translation.

Appearance changes: The design of the product's appearance changes in various ways depending on the internal structure of the product.

Components conversion: There are inevitably many furniture parts with massage function, so the design should be as clever as possible.

Size setting: The furniture has formed a fixed size. Health-care furniture needs to be more accurate according to different body groups, and divided into several commonly used sizes.

Massage intensity: "diarrhea" and "tonic" in acupoint massage refer to the intensity and method of massage. Different degrees of force achieve different effects and have a direct relationship to the prevention and treatment of diseases.

Length of time: it is a necessary condition for treating diseases, which needs to be considered when designing.

Temperature: The appropriate temperature can improve the treatment effect.

Avoid extreme populations: For example, elderly people with cervical spondylotic myelopathy, cervical spondylotic radiculopathy, fracture or joint damage, arteriosclerosis, hypertension, osteoporosis need careful massage. Patients with severe cardiovascular and cerebrovascular, acute and chronic infectious diseases, menstruation and pregnant, skin ulcer, spinal injuries should avoid massagers.

\section{Furniture of regimen based on acupuncts for chronic diseases}

\subsection{Acupoint dense area}

Massage furniture mainly includes: massage chairs, massage beds, and small massage devices for local use. According to the types of furniture in different use spaces, There are several types that are extracted from furniture that has a large contact area with the human body and has been used for a long time: beds, stools, chairs, sofas. Furniture of regimen is designed by combining acupuncture points with several commonly used furniture. The size can be divided into large, medium and small according to the average size of the human body to meet the needs of different tall, short, and fat people. The human body performs acupuncture therapy massage during daily use of furniture, and finally achieves the purpose of prevention and treatment. As shown in tables, designers can design furniture of 
regimen according to the distribution of acupoint-dense areas on furniture. Through these areas, the health effect can be maximized scientifically and the purpose of this article is to be studied.

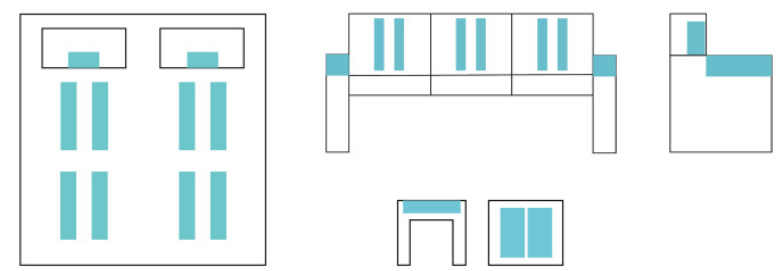

Figure. 7 Acupoint dense area

\subsection{A machine with a grid-like massage path}

There are four bladder meridians on the back of the human body, corresponding to four vertical working lines of a grid-like path. Common points for treating diseases can be found in square areas. As the picture shows, the concentric circles in the picture are manipulators (massage points). Different manipulators can be controlled for targeted treatment for different diseases. Start all the manipulators can massage the diseased area to the greatest extent; The manipulator can massage four bladder meridians up and down at the same time, half of the manipulator can be retracted for alternating left and right massages. If you need to massage the thoracic vertebra and waist, you can start local massage; The top of each manipulator has a device that can heat, vibrate, rotate itself and tap to cover more points and improve the treatment effect.
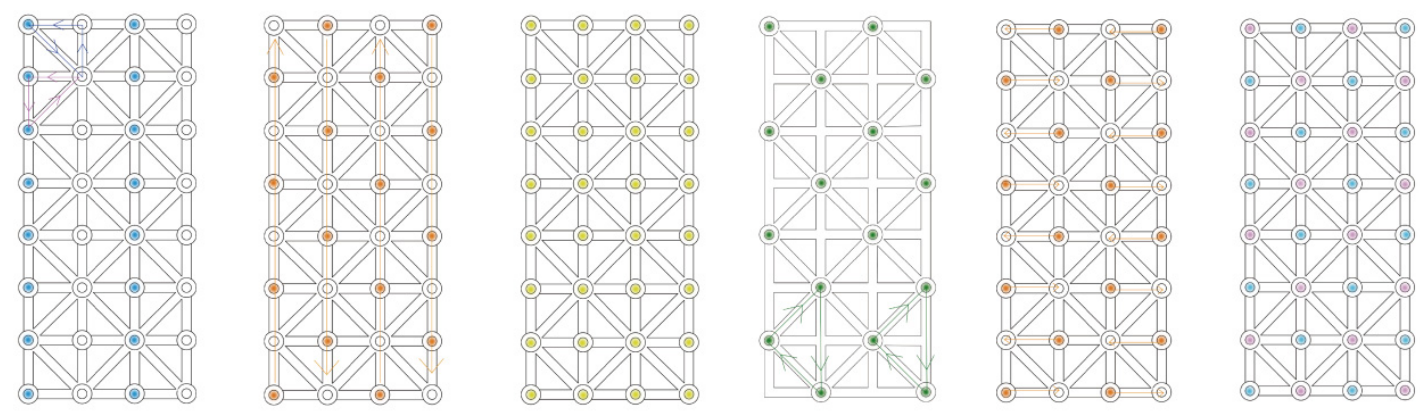

Figure. 8 A machine with a grid-like massage paths

\section{Summary}

Modern people are gradually paying attention to their body. After research in this article, we found that more than half of the chronic diseases can be prevented in daily life. Furniture is the longest time in the items we come in contact with daily. Through the acupoint-dense areas and grid-like massage paths extracted from this article, designers can use it in the design of furniture to achieve the effect of auxiliary treatment or prevention. In addition, for the entire furniture industry, the furniture of regime is a new direction that can be broken through and it is an inevitable way to improve the use of furniture.

\section{Acknowledgments}

This work was financially supported by "Thirteenth FiveYear Plan" national key research and development plan project (2018YFD0600300), the central financial support for local science and technology development projects (2019CT5008) and Hunan Forestry Science and Technology Innovation Project (XLK201946).

\section{References}

1. Wang,H. (2017) Effects of Moxibustion on clinical symptoms and renal hemodynamics in the patients with CKD 1-3.Zhejiang University of Traditional Chinese Medicine.D.

2. Chen D., Shen LM., Yu N.(2017) Study on the Development of Massage Theory and Massage Furniture.J.Furniture and interior

3. Tan S. (2013) The clinical observation on acupuncture for treatment of rheumatoid arthritis of notifying kidney and dispelling the blood stasis.D.Hubei University of Traditional Chinese Medicine

4. Wang Q., Duan C. Acupoint application therapy for winter disease and summer treatment and TCM health.D.Beijing university of chinese medicine

5. Guo S., Shi L.,Ji K.,Wang L.(2019) Investigation on Epidemiological Characteristics of Acupoint Application Therapy for Winter Disease and Summer Treatment in Respiratory 
Diseases.J.Journal of Liaoning University of Traditional Chinese Medicine

6. Li J., Hhuang G., Zhang X.(2010)Compound clove appetizer sticking Shenque acupoint to treat chronic heart failure Observation of curative effect in patients with abdominal distension.J.Modern Clinical Nursing:15-16

7. Li L. (2007) Coronary heart disease and angina pectoris.D.Beijing university of chinese medicine

8. Shen E.(2002) Governor of new style health care furniture abroad.J.Appliances:64-65

9. Duan Y. (2010) Discussion and Experimental Study of Heyuhui Acupoint Therapy for Liver Fibrosis.D.Guangzhou university of chinese medicine

10. Zhang K.,Xu Y.,Ding S.,Hong S.(2017)Study on Selecting Points of Acupuncture for Rheumatoid Arthritis Based on Literature.J.Chinese Acupuncture:221-224

11. Yan C.(2016) Clinical Effect and Mechanism of Acupoint Application of "Winter Disease and Summer Treatment" Based on Plasma Metabolomics in Treating Chronic Bronchitis in Remission.D.Hebei Medical University

12. Wei J.,Wang C.,Tang L.,Mao Q.(2019)Study on the Effect of Meridian Health Care in the Last 5 Years.J.Jiangxi Traditional Chinese Medicine:71-74

13. Zhang G.(2018) Research on Home-Based Rehabilitation Furniture Design for the Elderly.D.Nanjing Arts Institute

14. He Z. (2013)Clinical study on the treatment of angina pectoris with coronary heart disease.D.Guangzhou university of chinese medicine

15. Wei Q. (2009) Talking about the innovative design of health furniture.J.Art appreciation: 98-99

16. Zhang R.(2011) Clinical Observation on Acupoint Application Combined with Modified Yougui Bushen Decoction in Treating Chronic Aplastic Anemia with Spleen-Kidney Deficiency Syndrome.D.Hunan university of chinese medicine

17. Wang H.(2011) Clinical Observation on Acupoint Moxibustion for Leukopenia Caused by Chemotherapy.D.Beijing university of chinese medicine

18. Xu j.(2012) Clinical Study on Acupoint Application of Zhiji Zhushui Cream in Treating Cirrhosis and Ascites.D.Heilongjiang Institute of Traditional Chinese Medicine

19. Zheng W.(2010) Development of Wuyi Health Tourism Products.D.East China Normal University

20. Pan Y. (2011) Research Progress in Traditional Chinese Medicine of Primary Nephrotic Syndrome.D.Beijing university of chinese medicine

21. Liu W.(2008) Experimental study on the effect of acupuncture at different meridian points on blood pressure in spontaneously hypertensive rats.D.Beijing university of chinese medicine
22. Zhang J. (2016) Clinical Study on Acupuncture Points for Gastrointestinal Dysfunction of Chronic Liver Disease.D.Hubei university of chinese medicine

23. Li L.,Chen Y. (2011) Clinical research progress of acupuncture treatment of rheumatoid arthritis.J.Shanghai Journal of Acupuncture:243-246

24. Wang C.,Wang X.,Yu D.,Zhuang S.,Dong Z.,Shi J. (2017) Analysis of Epidemic Trends of Chronic Diseases in Coastal Areas of Eastern China.J.Chinese Public Health:1563-1566

25. Zhang L. (2016) Research progress on acupoint application of traditional Chinese medicine for chronic kidney disease and its complications.J.Hunan Journal of Traditional Chinese Medicine:190-192

26. Zhang Y. (2019) TCM chronic disease management and TCM nursing technology.J.Shanghai Nursing:71-75

27. Liu J.,He D. (2014) Research progress in external treatment of traditional Chinese medicine for rheumatoid arthritis.J.Research progress in external treatment of traditional Chinese medicine for rheumatoid arthritis.J.Rheumatism and arthritis

28. Hu Z.,Yin G. (1999) Effects of acupuncture on T cell subsets and IL-4 in patients with bronchial asthma.J.Chinese Acupuncture: 111-112

29. Zhao Z. (2013) Observation on the effect of acupoint application of winter disease and summer treatment on chronic asthma.J.Hubei Journal of Traditional Chinese Medicine

30. $\mathrm{Hu}$ P. (2013) Clinical Observation on Acupoint Application for Chronic Bronchitis.J.Practical Chinese Medicine: 50-51

31. Zhao G.,Zhang H. (2009) Observation on the curative effect of acupuncture treatment on aplastic.J.Chinese Minkang Medicine: 2235

32. Yang Z.,Zhang L.,Gu X. (2009) Clinical Observation on 36 Cases of Aplastic Ane 